\title{
LIVER CANCER
}

\section{Different effects of the Notch receptors in liver cancer revealed}

The various Notch receptors can have very different roles in the development of liver cancer, reports a new study in Hepatology. Furthermore, it is suggested that therapy based on anti-Notch 2 antibodies could be used for the treatment of patients with liver cancer.

The Notch signalling pathway seems to be involved in the development of liver cancer, but the exact details of this involvement are unknown. In order to elucidate the role of the different components of the pathway, the research team used antibodies to specifically target Notch 1, Notch 2, Notch 3 or Jag 1 in a mouse model of primary liver cancer driven by AKT and NRas.

Inhibiting Notch 2, or its ligand Jag 1, resulted in fewer tumours developing in the mice. Inhibition of Notch 3 had no effect on tumour burden. Interestingly, inhibiting Notch 1 reduced the number of hepatocellular-carcinoma-like tumours but increased the amount of cholangiocarcinoma-like tumours. In addition, Notch 2 and Jag 1 were expressed in human samples of hepatocellular carcinoma, and Notch 2 signalling was activated.

The clear involvement of Notch 2 led the team to investigate whether targeting Notch 2 was an effective cancer therapy. Antibody treatment to inhibit Notch 2 or Jag 1 was started either 2 or 3 weeks after the initiation of tumour development in mice, which resulted in a reduced tumour burden. Central necrosis was observed in the tumours of the mice that started treatment at 3 weeks, suggesting that the intervention affected established tumours.

\section{Claire Greenhill}

Original article Huntzicker, E. G. et al. Differential effects of targeting Notch receptors in a mouse model of liver cancer. Hepatology doi:10.1002/hep.27566 